\title{
The evolutionary development of the renin angiotensin aldosterone system and its importance for the survival of the human species
}

\author{
Natalia Butt-Hussaim, Jacek Manitius \\ Department of Nephrology, Hypertension and Internal Diseases, Dr. A. Jurasz University \\ Hospital No. 1 in Bydgoszcz, Nicolaus Copernicus University in Toruń, Ludwik Rydygier Collegium Medicum in Bygoszcz, Poland
}

\begin{abstract}
Kidneys produce a number of substances that affect intrarenal blood circulation; however, the key system that regulates blood flow in both general and local circulation (including the renal circulation) is the renin-angiotensinaldosterone system (RAAS). Individual elements of the RAA system are synthesized in separate tissues of the body under the influence of specific local factors. The system functions as a whole due to mutual relations based on feedback and it consists of three basic elements: renin, angiotensin and aldosterone.

The history of research on the RAA system dates back to the late $19^{\text {th }}$ century. One of the important stages of exploring the mechanisms related to RAA system functioning was the publication (in 1898) of the results of research on the hypertensive effect on blood pressure of rabbit kidney extracts (containing renin). The observations from 1934 were of similar significance: the correlation between dog kidney ischaemia and the occurrence of hypertension was found. In the following years, the enzymatic properties and structure of renin and angiotensin peptides, resulting from the action of renin and the enzyme converting angiotensin I (Ang I) to its active form — angiotensin II (Ang II), were clarified. The latter belongs to the most important regulators of aldosterone secretion. In 1939, it was proved that under the influence of renin blood pressure-rising peptides are formed. Consequently, it was documented that angiotensin was the cause of hypertension in animals with ischaemic kidney, and in 1954 the sequence of angiotensin I and II was described. In 1960-1961 systemic RAA occurrences were identified.

However, to provide the insight of evolutionary significance of the RAA system for humans, the phylogenetic development of this enzyme-endocrine system in vertebrates should be investigated. The largest database of information regarding this system in the aforementioned group of animals is the research of Hirofumi Sokabe and Hiroko Nishimura, which, among others, is the basis for this manuscript.
\end{abstract}

Key words: renin; angiotensin; angiotensin; aldosterone; vertebrates

Arterial Hypertens. 2021, vol. 25, no. 1, pages: 1-6 DOI: 10.5603/AH.a2020.0021

Address for correspondence: Natalia Butt-Hussaim, Department of Nephrology, Hypertension and Internal Diseases, Dr A. Jurasz University, Hospital No. 1 in Bydgoszcz, Nicolaus Copernicus University in Toruń Ludwik Rydygier Collegium Medicum in Bygoszcz; e-mail: nabuhu@gmail.com

This article is available in open access under Creative Common Attribution-Non-Commercial-No Derivatives 4.0 International (CC BY-NC-ND 4.0) license, allowing to download articles and share them with others as long as they credit the authors and the publisher, but without permission to change them in any way or use them commercially 


\section{Introduction}

Kidneys produce a number of substances that affect intrarenal blood circulation; however, the key system that regulates blood flow in both general and local circulation (including the renal circulation) is the reninangiotensin-aldosterone system (RAAS). Individual elements of the RAA system are synthesized in separate tissues of the body under the influence of specific local factors. The system functions as a whole due to mutual relations based on feedback and it consists of three basic elements: renin, angiotensin and aldosterone [1-3].

The history of research on the RAA system dates back to the late 19th century. One of the important stages of exploring the mechanisms related to RAA system functioning was the publication (in 1898) of the results of research on the hypertensive effect on blood pressure of rabbit kidney extracts (containing renin) [4] obtained by prof. Robert Tigerstedt and his assistant Per Bergman. Goldblatt observations from 1934 were of similar significance. He found a correlation between dog kidney ischaemia and the occurrence of hypertension [2]. In the following years, the enzymatic properties and structure of renin and angiotensin peptides, resulting from the action of renin and the enzyme converting angiotensin I (Ang I) to its active form — angiotensin II (Ang II), were clarified. The latter belongs to the most important regulators of aldosterone secretion (discovered by Simpson, Tait and Wetstein in 1953). In 1939, Braun-Menandez and Page proved that under the influence of renin blood pressure-rising peptides are formed. Consequently, it was documented that angiotensin was the cause of hypertension in animals with ischaemic kidney, and in 1954 Skeggs described the sequence of angiotensin I and II. In 1960-1961, Davis, Genest, Laragh and others identified systemic RAA occurrences.

However, to provide the insight of evolutionary significance of the RAA system for humans, the phylogenetic development of this enzyme-endocrine system in vertebrates should be investigated. The largest database of information regarding this system in the aforementioned group of animals is the research of Hirofumi Sokabe and Hiroko Nishimura, which, among others, is the basis for this manuscript.

\section{Evolution of RAA system in vertebrates}

\section{Structure of the juxtaglomerular apparatus in vertebrates}

In the kidneys of mammals, the juxtaglomerular apparatus consists of:
- renin-secreting granular epithelial cells in the central part of the afferent glomerular arterioles;

- macula densa composed of specialized cells originating from epithelial cells of the closer part of the distal tubule;

- extraglomerular mesangial cells;

- sympathetic nerves, densely innervating the afferent glomerular arterioles [6].

The structure of granular cells evolves at various stages of phylogenesis. In primitive vertebrates, i.e. jawless fishes, elasmobranches, holocephali, primary bony fishes, the structure and development of granular cells is different than in higher vertebrates. Granular cells in fish are localized externally to the afferent and efferent arterioles, whereby renin is secreted interstitially and enters the lymphatic circulation. In amphibians, reptiles and birds, granular cells occur in the area of the juxtaglomerular apparatus. In addition, the avian kidney has dense macular cells that are characteristic of macula dense in mammals. In mammalian kidneys, reninsecreting cells are present in the efferent arterioles; however, their number is smaller than in the afferent arterioles. As for the vascular component of the juxtaglomerular apparatus, it evolved earlier than the macula densa [6].

There is a well-described sympathetic innervation of glomerular cells and afferent arterioles in the mammalian group, unlike primitive vertebrates. In the arterioles of the batrachoidiformes, despite the abundant presence of renin-secreting cells, sympathetic nerve fibres have not yet been determined. In amphibians and birds, similarly as in mammals, the presence of nerve fibres has been confirmed histochemically and ultrastructurally.

\section{Biochemical and molecular structure of RAA system in vertebrates}

\section{Angiotensinogen and renin}

The occurrence of various forms of Ang I and Ang II in vertebrates (including the primary forms of Ang I and Ang II in elasmobranches) suggests the existence of many forms of angiotensinogen. Angiotensinogen concentrations, determined by maximum Ang I plasma production, are higher in higher vertebrates. In contrast, the ability to produce renin was found in all representative vertebrate species. There is a view that the RAA system, or its major components, developed in the early stages of vertebrate evolution. Unfortunately, the molecular identification of renin is significantly limited in primary vertebrates [5]. 


\section{Angiotensin}

Primary Ang I (decapeptide) and Ang II (octapeptide) are found in all vertebrates. The variability of the primary structure of Ang II in the course of phylogenesis relates to amino acids in position I (Asn, Asp, Tyr or additional chain), 3 (Val, Ile, Pro), 4 (His) and 5 (Ile, Val). Asn1AngII is the original form of Ang II. Changes at the first and ninth amino acid positions of Ang I result in a decrease in contractile activity, as does the removal or change of the first amino acid of Ang II [5].

\section{Angiotensin-converting enzymes and angiotensinases}

There are two biologically active ACEs in vertebrates, i.e. ACE 1 and ACE 2. Angiotensin converting enzymes are found in plasma, lungs, kidneys, gills, brain and other tissues of various vertebrate species, including lampetra fluviatilis, separate the last two amino acids of angiotensin (in vitro). Angiotensinase activity was also found in plasma, kidneys and other tissues in non-mammalian vertebrates [5].

\section{Angiotensin receptors}

Angiotensin AT1 and AT2 receptors were detected in only few non-mammalian vertebrate species. The first primary vertebrate belonging to agnatha superclass, in which angiotensin, ACE, and angiotensin receptors were found, is lampetra fluviatilis [5].

\section{Tissue RA}

The tissue renin angiotensin system exerts autocrine or paracrine effects in vertebrates, and the whole system or its components are found in the adrenal glands (adrenal tissue) and heart [5].

\section{Function and regulation of RAA system in vertebrates}

Mammals have four main mechanisms to control renin secretion:

- intrarenal baroreceptors that detect changes in blood pressure in the renal artery or nearby glomerular cells;

- a macula densa in the proximal part of the distal tubule, detecting the speed of $\mathrm{NaCl}$ ionic transport and transmitting information via specific mediators to the juxtaglomerular cells;

- sympathetic conduction via $\beta$-adrenoreceptors;

- many other, various humoral factors, including prostanoids, angiotensin, atrial natriuretic peptide (ANP), nitric oxygen (NO) and other mechanisms (no evidence of their occurrence in teleostei) [5].

Renal artery baroreceptors due to reduced blood pressure, also in fish, reptiles and birds, secondarily lead to a significant increase in plasma renin activity.

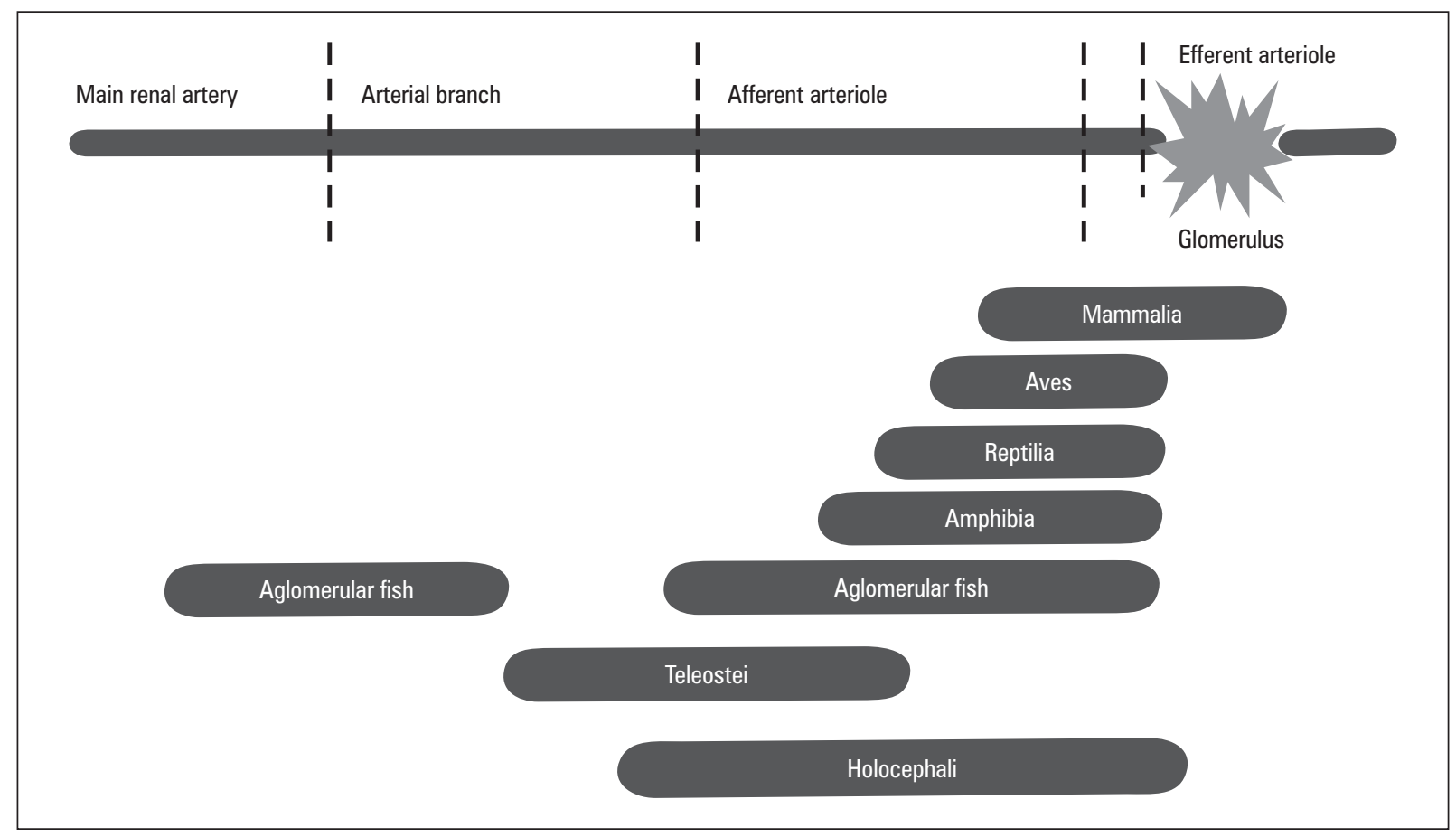

Figure 1. Distribution of granular cells along the arteries and arterioles of the kidneys in vertebrates. During the progress of phylogenesis in vertebrates, granulosa cells gradually 


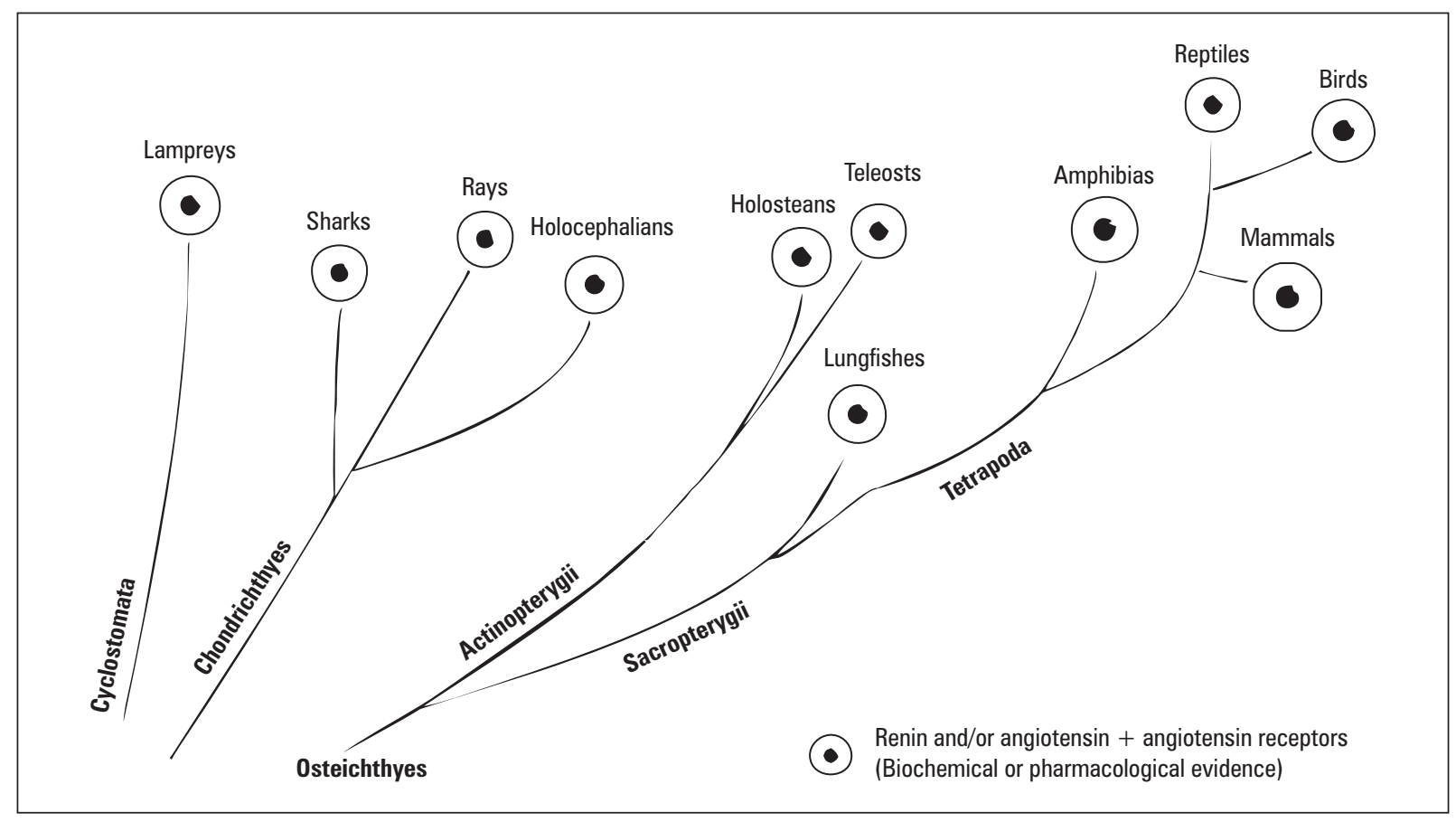

Figure 2. Renin/angiotensin and angiotensin receptors presence in vertebrates

This means that the lack or incomplete juxtaposition apparatus in lower vertebrates has been replaced by a renal mechanism of activating baroreceptors to control renin secretion. The exception are birds in which much less activity of the renin-angiotensin system in sudden onset of hypotension or hypovolemia is observed. This is due to the unique mechanism of rapid blood volume replenishment by absorbing fluid from interstitial tissue into the vessels.

Referring to macula densa mechanism, an increase in $\mathrm{NaCl}$ concentration in its cells causes an increase in the afferent arteriole resistance in a few seconds, followed by a reduction in glomerular filtration and a decrease in capillary glomerular pressure. This negative feedback that stabilizes the glomerular filtration is caused by a decrease in renin secretion by the juxtaglomerular cells. Although the renin-angiotensin system is only a negative feedback modulator, a decrease in renin secretion may lead to a decrease in salt concentration and reabsorption of water in the proximal tubule. The role of central baroreceptors and sympathetic nervous system in lower vertebrates is still being studied [5].

\section{Biological effects of angiotensin in vertebrates}

Ang II increases blood pressure in individual vertebrate species by increasing peripheral vascular resistance, indirectly, by releasing catecholamines and by central activity. In the most primitive vertebrates, the vasopressor effect of Ang II (Asn1, Val5) occurs indirectly by releasing catecholamines by stimulating sympathetic nerve endings or ganglia. Among teleostei, amphibians, reptiles and birds Ang II (Asp1) or (Asn 1$)$ increases blood pressure depending on the amount of angiotensin-dependent vasoreceptors. The effect of Ang II on catecholamine secretion by the sympathetic nervous system, adrenal medullary cells or chromophilic cells was developed in early phylogenesis.

Lower vertebrate kidneys are not or are only slightly self-regulated — changes in blood pressure in the aorta easily affect glomerular filtration and renal blood flow, which seems to be a mechanism controlling fluid and electrolyte excretion. Even if the birds evolved from ancient reptiles and descended from a line leading to mammals, the vascular effect of avian Ang II is significantly different from that of reptiles and mammals. The stimulated by Ang II vasopressor effects in adult birds is attributed solely to the stimulation of secretion of catecholamines from the adrenal medulla and adrenergic nerve endings.

Ang II in mammals stimulates aldosterone production in the early and late stages of steroidogenesis, by stimulating the conversion of cholesterol to pregnenolone and by stimulating the synthesis of active aldosterone. Similarly, to the concentration of potassium ions, it is an important regulator of aldosterone secretion. In anamnia belonging to vertebrates, such as elasmobranches, bony fishes, anurana 
and caudate amphibians, the adrenal cortex and medulla are located in and on the abdominal surface of the kidney (this is more of the adrenal gland than the adrenal cortex). In contrast, reptiles and birds have discreetly separated the adrenal glands into the adrenal cortex and medulla area, but it is not as visible as in mammals.

In elasmobranches, the main corticosteroids in the intrarenal tissue and circulation are 17-hydroxylase $1 \alpha$-hydroxycorticosterone, while in teleostei cortisol is the quantitatively dominant steroid hormone synthesized in the intrarenal tissue and has mineralocorticosteroid activity. Alderone and corticosterone occur in amphibians, and corticosterone is the main adrenal steroid in reptiles. Among birds, Ang II stimulates the synthesis of aldosterone in the adrenal glands [5].

\section{Conclusion}

It is believed that each component of the juxtaglomerular apparatus developed at a different stage of phylogenesis. Granular cells producing renin or renin-like enzymes developed earlier, while macula dense and extraglomerular mesangial cells appeared in higher vertebrates. It is unclear when abundant adrenergic innervation appeared in the juxtaglomerular cells (at the level of teleostei there is no evidence of communication between renin-secreting cells and nerves or adrenergic receptors). The distribution and location of the juxtaglomerular cells seem to have been shifted during phylogenesis.

In primitive vertebrates, renin-secreting cells are scattered along the small arteries and arterioles in the kidney. Along with the phylogenetic advancement of vertebrates, renin-secreting cells moved to the area of the juxtaglomerular apparatus. The presence of prorenin and active renin as well as its regulatory mechanisms are probably the remains of non-mammalian vertebrates.

It is a fact that angiotensin is present in all vertebrate species. Primary Ang II is a stable molecule that occurs during phylogeny, with variation in amino acids at positions 1, 3, 4 and 5. Lampetra fluviatilis, similarly to teleostei, has Ang II of a unique structure. AT receptors are found in most vertebrates (the problem with the determination of receptors in lower vertebrates is related to the different structure of the junction site with Ang). The degree of similarity increases along with the development of vertebrates and suggests that all AT1 receptor homologues can derive from the same protoplast. Unfortunately, there is no confirmed data on the simultaneous evolution of the angiotensin receptor with the development of the RA system (under study).

The relationship between the RA system and steroid hormones of the adrenal glands occurs in every class of vertebrates, except for jawless fishes. The adrenal tissue of reptiles and birds is defective, only in mammals it is complete and has a layer producing glyco- and corticosteroids. Ang II stimulates the release of the production of $1 \alpha$-hydroxycorticosterone (elasmobranches), cortisol (teleostei) and corticosterone/aldosterone (amphibians, reptiles, birds). Adrenocorticotropic hormone $(\mathrm{ACTH})$ is less often the main regulator of adrenal (intrarenal) hormones than Ang II. The role of mineralocorticoids, including aldosterone, in sodium renal tubule transport is unclear in nonmammalian vertebrates.

Summarizing the evolutionary aspects of RAA system development in individual animal classes, Ang II causes an increase in blood pressure in representative vertebrate species directly through vascular smooth muscle spasm or indirectly by the secretion of catecholamines from the adrenergic nerve endings and the adrenal medulla/chromatophilic cells. Haemorrhage or pharmacologically induced hypotension causes an increase in renin secretion or the production of angiotensin.

Maintaining normal blood pressure can be one of the fundamental roles of the RAA system in human. In mammals, including humans, an immediate response to dramatic drops in blood pressure or volume occurs in a neurological mechanism through baroreceptors that stimulate the RAA system to restore blood pressure. Long-term recovery or maintenance of blood pressure and volume is constantly controlled by the kidneys. In addition, it is even more complex due to the fact that apart from the systemically acting RAA, there are also local counterparts, acting in such tissues as the brain, heart, blood vessel walls or kidneys, causing an increase in blood pressure.

Unfortunately, during long-term stimulation of RAA, Ang II systemically increases the synthesis and secretion of aldosterone as well as growth factors and pro-inflammatory cytokines, causing cardiovascular remodelling along with hypertension leading to secondary damage to such organs as heart or kidneys. Locally acting Ang II (in tissues) has a mitogenic effect on cardiac myocytes and vascular smooth muscle cells; it also increases collagen synthesis in the myocardium and vessels, contributing to the development of left ventricular hypertrophy, thickening of the arterial walls and end organ damage. 
At the end of the discussion, it should be stated that the RAA system, which in the course of evolutionary development protected against a decrease in blood pressure, is currently the reason for the excessive persistence of increased blood pressure values and secondary organ damage. For this reason, the inclusion of drugs that inhibit RAA components is currently the primary treatment for cardiovascular and renal diseases.

\section{References}

1. Cowley AW. Long-term control of arterial blood pressure. Physiol Rev. 1992; 72(1): 231-300, doi: 10.1152/physrev.1992.72.1.231, indexed in Pubmed: 1731371.
2. Goldblatt H, Lynch J, Hanzal RF, et al. Studies on experimental hypertension : I. The production of persistent elevation of systolic blood pressure by means of renal ischemia. J Exp Med. 1934; 59(3): 347-379, doi: $10.1084 / \mathrm{jem} .59 .3 .347$, indexed in Pubmed: 19870251.

3. Guyton AC. Blood pressure contro - special role of the kidneys and body fluids. Science. 1991; 252(5014): 18131816, doi: 10.1126/science.2063193, indexed in Pubmed: 2063193.

4. Marks LS, Maxwell MH. Tigerstedt and the discovery of renin. An historical note. Hypertension. 1979; 1(4): 384-388, doi: 10.1161/01.hyp.1.4.384, indexed in Pubmed: 396241.

5. Nishimura H. Renin-angiotensin system in vertebrates: phylogenetic view of structure and function. Anat Sci Int. 2017; 92(2): 215-247, doi: 10.1007/s12565-016-0372-8, indexed in Pubmed: 27718210.

6. Sokabe H. Phylogeny of the renal effects of angiotensin. Kidney Int. 1974; 6(5): 263-271, doi: 10.1038/ki.1974.112, indexed in Pubmed: 4372451. 